\title{
Optimal Control of a Three-Body Hinge-Barge Wave Energy Device Using Pseudospectral Methods
}

\author{
Francesco Paparella and John V. Ringwood, Senior Member, IEEE
}

\begin{abstract}
This paper shows the potential of optimal control for enhancing the power capture for a three-body hinge-barge wave energy converter (WEC). It is the first documented application of a coordinated control strategy for a hinge-barge WEC, where power is converted at the joints between both the fore and aft pontoons, and the central barge section. Two separate optimal control formulations, based on different model representations, are evaluated and are shown to considerably outperform an optimal constant damping control strategy.
\end{abstract}

Index Terms-Model-based control, multi-body wave energy converters (WECs), pseudo-spectral (PS) methods.

\section{INTRODUCTION}

$\mathbf{H}$ INGE-BARGE wave energy converters (WECs) are articulated floating structures that extract energy carried by the waves. The hinge-barge device is composed by a number of rectangular bodies interconnected by hinges, and it is considered to be an attenuator device since it operates longitudinally to the direction to the incoming wave. The relative pitch motion between each pair of bodies is used to drive a Power Take Off (PTO) system. Examples of hinge-barge WECs include the McCabe Wave Pump [1] and the SeaPower Platform [2]. Another example of an articulated WEC is the Pelamis WEC which is composed by multiple cylindrical section linked by hinged joints [3]. For the Pelamis WEC, the control of the power absorbed at each joint axis is realized considering the inputs from all axes. Therefore, the real-time control of all forces is realized with respect to the entire machine response. However, little detail on the control strategies for the Pelamis WEC is provided in the available literature. In [4], a hinged five-body WEC consisting of a circular center floater hinged to four smaller spherical buoys is considered. The relative rotation between the central body and each buoy is used to drive a PTO, and the absorbed energy is maximized for both regular and irregular waves. For regular waves, the optimal velocities and control forces are computed at each frequency of the incident wave for both passive and active control. The optimization of the damping coefficients of passive PTOs is also considered for the regular wave case. For irregular waves, only the optimization of frequency independent

Manuscript received February 1, 2016; revised May 11, 2016 and July 5, 2016; accepted July 13,2016. Date of publication July 18, 2016; date of current version December 14, 2016. This work was supported by the Science Foundation Ireland for the Marine Renewable Ireland Centre under Grant 12/RC/2302. Paper no. TSTE-00097-2016.

The authors are with the Centre for Ocean Energy Research, Maynooth University, Kildare, Ireland (e-mail: francesco.paparella.2014@mumail.ie; john.ringwood@eeng.nuim.ie).

Color versions of one or more of the figures in this paper are available online at http://ieeexplore.ieee.org.

Digital Object Identifier 10.1109/TSTE.2016.2592099 damping coefficients of passive PTOs is considered for waves represented by different realizations of the JONSWAP spectrum. In this paper, both passive and active controllers, which computes the optimal profile of velocities and control forces of the PTOs, is considered for both regular and irregular waves.

For the optimal control of WECs, the model predictive control (MPC) strategy has been adopted in [5]. More recently, an optimal causal control that does not require predictions of the future velocities of the WEC is proposed in [6]. The objective of this paper is to asses the value of optimal control with pseudospectral (PS) methods applied to a three-body hinge-barge wave energy device. The control is based on the model of the device, and it takes into account the hydrodynamic and kinematic interactions between the bodies. The PTO loads at the joints are controlled in a coordinated way, so that the total energy extracted by the device is maximized. In this paper, only the steady-state solution to the optimal control problem is considered, therefore the transient response of the device is neglected. An PS optimal controller that also considers the transient effects can also be employed [7].

Optimal control of the hinge-barge device is realized using PS methods, which are a subset of the class of techniques used for the discretisation of integral and partial differential equations known as mean weighted residuals [8], [9]. In [10], PS methods are applied for the optimal control of WECs. PS methods are used to transform the optimal control problem of the hinge-barge device into a finite dimensional optimization problem which can be nonconvex for both passive and active control.

The remainder of the paper is organized as follows: In Section II, the dynamic model of a three-body hinge-barge device is derived while, in Section III, PS methods are applied to solve the optimal control of a three-body hinge-barge device. In Section IV, PS methods are compared to a standard optimal controller for both monochromatic and polychromatic waves while, in Section V, a method to convexify the optimization problem obtained with PS methods is presented. Finally, overall conclusions are drawn in Section VI.

\section{Dynamic Model of A Three-Body HINGE-BARGE DEVICE}

This section describes the dynamic model of a three-body hinge-barge device originally derived in [11]. In Fig. 1, the device is represented together with the global frame $X_{g}, Z_{q}$, while a body frame is assigned to each body composing the device. The damping plate connected to body 2 aims to reduce the vertical motion of body 2, increasing the pitch motions of body 1 and 3 . The analysis of the motion of the devices is restricted to the two dimensional plane $X-Z$. The total 


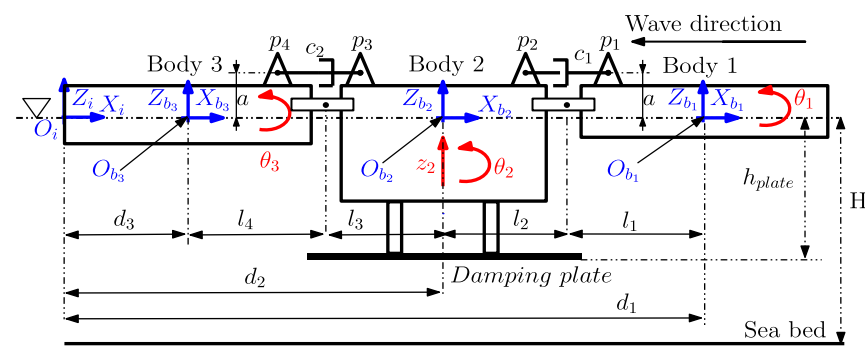

Fig. 1. Three-body hinge-barge device where $X_{g} Z_{g}$ represents the global frame, and a local frame is assigned to each body composing the device.

number of independent degrees of freedom of the system in Fig. 1 is four: The heave displacement $z_{2}$ of body 2 , and the pitch angles $\theta_{1}, \theta_{2}$ and $\theta_{3}$ of bodies 1,2 and 3 , respectively.

The linear dynamic model of the device can be derived with two different formulations: the differential and algebraic equations (DAEs) formulation and the ordinary differential equations (ODEs) formulation. In the DAE formulation, the model is described as a set of differential equations for the generalized coordinates of the unconstrained system and the constraints are described by algebraic equations. In the ODE formulation, the constraint equations are no longer described explicitly, but rather embedded into a set of differential equations for the independent degrees of freedom of the system only. Both formulations are retained in this paper, since different control solutions are obtained depending on which formulation is used to describe the dynamics of the device.

\section{A. DAE Formulation}

In this section, the DAE formulation is applied in order to obtain the equations of motion for a three-body hinge-barge device. The linearized equations of motion are given as follows [11]:

$$
\dot{\mathbf{q}}=\mathbf{v}
$$

$$
\begin{aligned}
\mathbf{M} \dot{\mathbf{v}}+\mathbf{B}_{\mathrm{visc}} \mathbf{v}+\mathbf{C}_{z}^{T} \boldsymbol{\lambda}= & -\mathbf{G q}-\mathbf{M}_{\infty} \dot{\mathbf{v}}+\cdots-\int_{-\infty}^{t} \mathbf{K}_{\mathrm{rad}} \\
& \times(t-\tau) \mathbf{v}, d \tau+\mathbf{f}_{\mathrm{wave}}+\mathbf{f}_{\mathrm{PTO}} \\
\mathbf{C}(\mathbf{z})= & 0
\end{aligned}
$$

where $\mathbf{z}$ is the vector of generalized positions expressed in the body frame of each body:

$$
\mathbf{z}=\left[\begin{array}{lll}
\mathbf{z}_{1} & \mathbf{z}_{2} & \mathbf{z}_{3}
\end{array}\right]^{T}=\left[\begin{array}{llllll}
z_{i, b_{1}}^{b} & \theta_{1} & z_{i, b_{2}}^{b} & \theta_{2} & z_{i, b_{3}}^{b} & \theta_{3}
\end{array}\right]^{T}
$$

where $z_{i, b_{k}}^{b}$ and $\theta_{k}$ are the heave displacement and pitch angle of body $k$, respectively, with $k=1,2,3$. The vector $\mathbf{v}$ represents the generalized velocities expressed in the body frame of each body, while the vector $\mathbf{q}$ represents the generalized positions expressed in the global frame.

The rigid-body inertia matrix $\mathbf{M}$ in equation (2) is a block matrix where each block $\mathbf{M}^{b_{k}}$ is given as

$$
\mathbf{M}^{b_{k}}=\left[\begin{array}{cc}
m_{k} & 0 \\
0 & I_{\mathrm{yy}, k}+m_{k} h_{g, k}^{2}
\end{array}\right]
$$

where $k=1,2,3, m_{k}$ is the mass of body $k, I_{\mathrm{yy}, k}$ is the moment of inertia of body $k$ around the $y$-axis and $h_{g, k}$ is the distance of the center of mass of body $k$ from point $O_{\mathrm{bk}}$ along the $z$-axis. The rigid-body inertia matrix $\mathbf{M}^{b_{k}}$ does not contain off-diagonal terms since that the body coordinate frame of each body is a principal axis coordinate system, and the vector defining the position of the centre of gravity with respect to the origin of the body frame is defined as follows:

$$
\mathbf{r}_{g}^{b_{k}}=\left[\begin{array}{ll}
0 & 0-h_{g, k}
\end{array}\right]^{T}
$$

The term $\mathbf{B}_{\mathrm{visc}}$ in equation (2) represents the viscous matrix. The hydrodynamic loads $\mathbf{G}, \mathbf{M}_{\infty}, \mathbf{K}_{\mathrm{rad}}$ and $\mathbf{f}_{\mathrm{wave}}$ in equation (2) are obtained by means of the boundary element software WAMIT [12]. The vector of PTO forces $\mathbf{f}_{\mathrm{PTO}}$ in equation (2) is given by the forces due to the PTO systems connecting body 2 to bodies 1 and 3. As shown in Fig. 1, each PTO is modeled as a linear dash-pot system. The vector of loads, due to the PTO systems acting on the device, is given as follows:

$$
\mathbf{f}_{\mathrm{PTO}}=-\left[\begin{array}{llllll}
0 & -F_{s 1} a & 0 & F_{s 1} a-F_{s 2} a & 0 & F_{s 2} a
\end{array}\right]^{T}
$$

where

$$
\begin{aligned}
& F_{s 1}=c_{1} \dot{l}_{1} \\
& F_{s 2}=c_{2} \dot{l}_{2}
\end{aligned}
$$

where $c_{i}, l_{i}$, with $i=1,2$, are the damping coefficients and length of the dash-pot system connecting body 2 to body 1 and 3 , respectively. The parameter $a$ represents the vertical distance between the line of action of the PTO force and the center-line of the device.

Now, the constraint equations $\mathbf{C}(\mathbf{z})$ in equation (3) are derived. The constraint equations introduced by the hinge between bodies 2 and 3 linearized around the equilibrium position are given as follows:

$$
\mathbf{R}_{b}^{i}\left(\theta_{2}\right)\left(\left[\begin{array}{c}
0 \\
z_{i, b_{2}}^{b}
\end{array}\right]+\left[\begin{array}{c}
-l_{3} \\
0
\end{array}\right]\right)-\mathbf{R}_{b}^{i}\left(\theta_{3}\right)\left(\left[\begin{array}{c}
0 \\
z_{i, b 3}^{b}
\end{array}\right]+\left[\begin{array}{c}
l_{4} \\
0
\end{array}\right]\right)=0
$$

where $l_{3}$ and $l_{4}$ are the distances of the hinge from $O_{b_{2}}$ and $\mathrm{O}_{b_{3}}$, respectively. The constraint equations introduced by the hinge between bodies 1 and 2 linearized around the equilibrium position are given as follows:

$$
\mathbf{R}_{b}^{i}\left(\theta_{1}\right)\left(\left[\begin{array}{c}
0 \\
z_{i, b_{1}}^{b}
\end{array}\right]+\left[\begin{array}{c}
-l_{1} \\
0
\end{array}\right]\right)-\mathbf{R}_{b}^{i}\left(\theta_{2}\right)\left(\left[\begin{array}{c}
0 \\
z_{i, b 2}^{b}
\end{array}\right]+\left[\begin{array}{c}
l_{2} \\
0
\end{array}\right]\right)=0
$$

where $l_{1}$ and $l_{2}$ are the distances of the hinge from $O_{b_{1}}$ and $O_{b_{2}}$, respectively. The linearized rotation matrices $\mathbf{R}_{b}^{i}$ are given as follows:

$$
\mathbf{R}_{b}^{i}\left(\theta_{k}\right)=\left[\begin{array}{cc}
1 & -\theta_{k} \\
\theta_{k} & 1
\end{array}\right]
$$

with $k=1,2,3$. The constraints in equation (10) force the global position of the hinge defined by the coordinates of body 2 to be equal to the global position of the hinge defined by the coordinates of body 3. Similar considerations can be made for the constraints in equation (11). The matrix of the partial 
derivatives of constraint equations (10) and (11) computed with respect to the generalized positions and linearized around the equilibrium position, is given as follows:

$$
\mathbf{C}_{z}=\left[\begin{array}{cccccc}
0 & 0 & 1 & -l_{3} & -1 & -l_{4} \\
1 & -l_{1} & -1 & -l_{2} & 0 & 0
\end{array}\right]
$$

\section{B. ODE Formulation}

The ODE formulation is now applied to obtain the equations of motion of a three-body hinge-barge device. The vector of independent velocities of the device is

$$
\mathbf{v}_{s}=\left[\begin{array}{llll}
\dot{\theta}_{1} & \dot{z}_{i, b_{2}}^{b} & \dot{\theta}_{2} & \dot{\theta}_{3}
\end{array}\right]^{T} \text {. }
$$

Given the matrix $\mathbf{C}_{z}$ from equation (13), the transformation matrix $\mathbf{P}$ used to express the relation between the vector of generalized velocities and independent velocities is given as follows:

$$
\mathbf{P}=\left[\begin{array}{cccc}
l_{1} & 1 & l_{2} & 0 \\
1 & 0 & 0 & 0 \\
0 & 1 & 0 & 0 \\
0 & 0 & 1 & 0 \\
0 & 1 & -l_{3} & -l_{4} \\
0 & 0 & 0 & 1
\end{array}\right]
$$

Using $\mathbf{P}$, the equations of motion of the device expressed with respect to the independent degrees of freedom are given as follows:

$$
\begin{aligned}
& \dot{\mathbf{q}}=\mathbf{v}_{s} \\
& \mathbf{M}_{s} \dot{\mathbf{v}}_{s}+\left(\mathbf{B}_{s}+\mathbf{B}_{\mathrm{visc}, s}\right) \mathbf{v}_{s}=-\mathbf{G}_{s} \mathbf{q}_{s}-\mathbf{M}_{\infty, s} \dot{\mathbf{v}}_{s}+\cdots \\
& \quad-\int_{-\infty}^{t} \mathbf{K}_{\mathrm{rad}, s}(t-\tau) \mathbf{v}_{s}, d \tau+\mathbf{f}_{\mathrm{wave}, s}+\mathbf{f}_{\mathrm{PTO}, s}
\end{aligned}
$$

where

$$
\begin{aligned}
\mathbf{M}_{s} & =\mathbf{P}^{T} \mathbf{M P} \\
\mathbf{B}_{s} & =\mathbf{P}^{T} \mathbf{B P}+\mathbf{P}^{T} \mathbf{M} \dot{\mathbf{P}}+\mathbf{P}^{T} \mathbf{M}_{\infty} \dot{\mathbf{P}} \\
\mathbf{B}_{\mathrm{visc}, s} & =\mathbf{P}^{T} \mathbf{B}_{\mathrm{visc}} \mathbf{P} \\
\mathbf{G}_{s} & =\mathbf{P}^{T} \mathbf{G P} \\
\mathbf{M}_{\infty, s} & =\mathbf{P}^{T} \mathbf{M}_{\infty} \mathbf{P} \\
\mathbf{K}_{\mathrm{rad}, s} & =\mathbf{P}^{T} \mathbf{K}_{\mathrm{rad}} \mathbf{P} \\
\mathbf{f}_{\mathrm{wave}, s} & =\mathbf{P}^{T} \mathbf{f}_{\mathrm{wave}} \\
\mathbf{f}_{\mathrm{PTO}, s} & =\mathbf{P}^{T} \mathbf{f}_{\mathrm{PTO}} .
\end{aligned}
$$

\section{PS OPTIMAL WEC CONTROL}

This section describes the direct transcription of the optimal control problem for a three-body hinge barge device [10]. For a generic WEC control problem, the vector of PTO forces in equation (2) is considered to be $\mathbf{f}_{\mathrm{PTO}}=\mathbf{F}_{p} \mathbf{u}$, where $\mathbf{F}_{p}$ is the configuration matrix and $\mathbf{u}$ is the vector of control variables [10]. The configuration matrix $\mathbf{F}_{p}$ is used in order to combine, in different ways, the manipulated inputs. Therefore, the configuration matrix $\mathbf{F}_{p}$ depends on the PTO structure used. For the case of a three-body hinge-barge device, $\mathbf{F}_{p}$ is given as

$$
\mathbf{F}_{p}=\left[\begin{array}{cccccc}
0 & 1 & 0 & -1 & 0 & 0 \\
0 & 0 & 0 & -1 & 0 & 1
\end{array}\right]^{T}
$$

and the vector of control variables is considered to be $\mathbf{u}=\left[\begin{array}{ll}\tau_{1} & \tau_{2}\end{array}\right]^{T}$, where $\tau_{1}$ is the torque applied by the PTO connecting body 2 and 1 , while $\tau_{2}$ is the torque applied by the PTO connecting body 2 and 3 . In contrast to the PTOs considered in Section II-A, the torque applied by each PTO is independent of the relative pitch velocity between the connected bodies.

The average power absorbed by the PTOs, over the time interval $[0, T]$, is given as

$$
J=\frac{1}{T} \int_{0}^{T} \mathbf{v}^{T} \mathbf{F}_{p} \mathbf{u} d t .
$$

The optimal control problem consists of computing the vector of PTO torques that maximize the cost function in equation (27), subject to the equations of motion (1)-(3) and (16) and (17), for the DAE and ODE formulations, respectively. Additional constraints on the applied torques and relative pitch velocity between the bodies can be considered in the optimal control formulation.

For the discretization of the control problem, positions and velocities that appear in the equations of motion obtained for the DAE and ODE formulations, and the control torques, can be approximated with a linear combination of basis functions. Given the periodic nature of the variables associated with the problem, zero mean trigonometric polynomials (truncated Fourier series) represent a sensible choice for the approximation of positions, velocities and control torques. Therefore, the $i$ th components of the position and velocity vectors, and the $p$ th components of the control vector, are given as follows:

$$
\begin{aligned}
q_{i}(t) \approx q_{i}^{M}(t) & =\sum_{k=1}^{M} x_{i, k}^{q, c} \cos \left(k \omega_{0} t\right)+x_{i, k}^{q, s} \sin \left(k \omega_{0} t\right) \\
& =\boldsymbol{\Phi}(t) \hat{\mathbf{x}}_{i}^{q} \\
v_{i}(t) \approx v_{i}^{M}(t) & =\sum_{k=1}^{M} x_{i, k}^{v, c} \cos \left(k \omega_{0} t\right)+x_{i, k}^{v, s} \sin \left(k \omega_{0} t\right) \\
& =\boldsymbol{\Phi}(t) \hat{\mathbf{x}}_{i}^{v} \\
\tau_{p}(t) \approx \tau_{p}^{M}(t) & =\sum_{k=1}^{M} u_{p, k}^{c} \cos \left(k \omega_{0} t\right)+u_{p, k}^{s} \sin \left(k \omega_{0} t\right) \\
& =\boldsymbol{\Phi}(t) \hat{\mathbf{u}}_{p}
\end{aligned}
$$

where $i=1, \ldots, 6 N$ and $i=1, \ldots, n$ for the DAE and ODE formulations, respectively, and $p=1,2$. The parameter $M$ is the order of expansion for the positions, velocities and control torques. The vector of the coefficients $\hat{\mathbf{x}}_{i}^{q}, \hat{\mathbf{x}}_{i}^{v}$ and $\hat{\mathbf{u}}_{p}$ of the approximated components of the position, velocity and control 
vectors, are given as follows:

$$
\begin{aligned}
\hat{\mathbf{x}}_{i}^{q} & =\left[\begin{array}{lllll}
x_{i, 1}^{q, c} & x_{i, 1}^{q, s} & . . & x_{i, M}^{q, c} & x_{i, M}^{q, s}
\end{array}\right]^{T} \\
\hat{\mathbf{x}}_{i}^{v} & =\left[\begin{array}{lllll}
x_{i, 1}^{v, c} & x_{i, 1}^{v, s} & . . & x_{i, M}^{v, c} & x_{i, M}^{v, s}
\end{array}\right]^{T} \\
\hat{\mathbf{u}}_{p} & =\left[\begin{array}{lllll}
u_{p, 1}^{c} & u_{p, 1}^{s} & . . & u_{p, N_{u}}^{c} & u_{p, M}^{s}
\end{array}\right]^{T}
\end{aligned}
$$

while the basis function vector $\boldsymbol{\Phi}(t)$ is given as follows:

$$
\boldsymbol{\Phi}(t)=\left[\cos \left(\omega_{0} t\right) \sin \left(\omega_{0} t\right) \ldots \cos \left(M \omega_{0} t\right) \sin \left(M \omega_{0} t\right)\right]^{T}
$$

where $\omega_{0}=2 \pi / T$ is the fundamental frequency. Given the linear nature of the model, the frequencies associated with the system variables (forces, displacements and velocities) are consistent with the frequencies in the excitation signal i.e. no harmonics or subharmonics are produced. By substituting the approximated velocities and control torques defined in equations (29) and (30), respectively, into the expression for the absorbed power defined in equation (27), the approximated average absorbed power is given as

$$
\begin{aligned}
J^{M} & =\frac{1}{T} \int_{0}^{T} \boldsymbol{\Phi}(t)^{T} \mathbf{X}^{V} \mathbf{F}_{p} \mathbf{U}^{T} \mathbf{\Phi}(t) d t \\
& =\frac{1}{2}\left(\hat{\mathbf{u}}_{1}^{T}\left(\hat{\mathbf{x}}_{2}^{v}-\hat{\mathbf{x}}_{4}^{v}\right)+\hat{\mathbf{u}}_{2}^{T}\left(\hat{\mathbf{x}}_{6}^{v}-\hat{\mathbf{x}}_{4}^{v}\right)\right)
\end{aligned}
$$

where

$$
\begin{aligned}
\mathbf{X}^{V} & =\left[\hat{\mathbf{x}}_{1}^{v}, \ldots, \hat{\mathbf{x}}_{n}^{v}\right] \\
\mathbf{U} & =\left[\begin{array}{ll}
\hat{\mathbf{u}}_{1} & \hat{\mathbf{u}}_{2}
\end{array}\right] .
\end{aligned}
$$

Equation (35) is a quadratic cost function in the expansion coefficients of the control torques and velocities. The derivatives of the $i$ th components of the position and velocity vector are, respectively,

$$
\begin{aligned}
& \dot{q}_{i}^{M}(t)=\dot{\boldsymbol{\Phi}}(t)^{T} \hat{\mathbf{x}}_{i}^{q}=\boldsymbol{\Phi}(t)^{T} \mathbf{D}_{\phi} \hat{\mathbf{x}}_{i}^{q} \\
& \dot{v}_{i}^{M}(t)=\dot{\boldsymbol{\Phi}}(t)^{T} \hat{\mathbf{x}}_{i}^{v}=\boldsymbol{\Phi}(t)^{T} \mathbf{D}_{\phi} \hat{\mathbf{x}}_{i}^{v}
\end{aligned}
$$

where $\mathbf{D}_{\phi} \in \mathbb{R}^{2 M \times 2 M}$ is a block diagonal matrix, with the $k$ th block $(k=1, \ldots, M)$ given as follows:

$$
\mathbf{D}_{\phi, k}=\left[\begin{array}{cc}
0 & k \omega_{0} \\
-k \omega_{0} & 0
\end{array}\right] \text {. }
$$

Regarding the DAE formulation, substituting the approximated states (28), (29) and their time derivatives (38), (39) into the equations of motion (1)-(3) yields the following equations of motion in residual form:

$$
\begin{aligned}
r_{i}^{q}(t)= & \boldsymbol{\Phi}(t) \mathbf{D}_{\phi} \hat{\mathbf{x}}_{i}^{q}-\sum_{p=1}^{6 N} J_{i, p} \boldsymbol{\Phi}(t) \hat{\mathbf{x}}_{p}^{v} \\
r_{i}^{v}(t)= & \sum_{p=1}^{6 N} M_{i, p} \boldsymbol{\Phi}(t) \mathbf{D}_{\phi} \hat{\mathbf{x}}_{p}^{v}+\sum_{p=1}^{6 N} B_{i, p} \boldsymbol{\Phi}(t) \hat{\mathbf{x}}_{p}^{v}+\sum_{p=1}^{6 N} G_{i, p} \boldsymbol{\Phi}(t) \hat{\mathbf{x}}_{p}^{q} \\
& \quad+\sum_{p=1}^{6 N} \int_{-\infty}^{t} K_{\operatorname{rad}_{i, p}}(t-\tau) \boldsymbol{\Phi}(\tau) \hat{\mathbf{x}}_{p}^{v} d \tau+\sum_{p=1}^{m} C_{q_{i, p}}^{T} \\
& \times \mathbf{\Phi}(t) \hat{\mathbf{x}}_{p}^{\lambda}(t)-f_{\text {wave }, i}(t)-\sum_{p=1}^{2} F_{p_{i, p}} \boldsymbol{\Phi}(t) \hat{\mathbf{u}}_{p}
\end{aligned}
$$$$
r_{j}^{C}(t)=C_{j}(\mathbf{q}, t)
$$

where $i=1, \ldots, 6 N, j=1, \ldots, m$, and $J_{i, p}, M_{i, p}, B_{i, p}, G_{i, p}$, $K_{\mathrm{rad}_{i, p}}, C_{q_{i, p}}^{T}$ and $F_{p_{i, p}}$ are the elements of the matrices $\mathbf{J}(\boldsymbol{\Theta})$, $\mathbf{M}, \mathbf{B}, \mathbf{G}, \mathbf{K}_{\mathrm{rad}}, \mathbf{C}_{q}^{T}$ and $\mathbf{F}_{p}$ respectively. Regarding the ODE formulation, substituting the approximated states (28), (29) and their time derivatives (38), (39) into the equations of motion (16) and (17), yields the following equations of motion in residual form:

$$
\begin{aligned}
r_{i}^{q}(t)= & \boldsymbol{\Phi}(t) \mathbf{D}_{\phi} \hat{\mathbf{x}}_{i}^{q}-\boldsymbol{\Phi}(t) \hat{\mathbf{x}}_{i}^{v} \\
r_{i}^{v}(t)= & \sum_{p=1}^{n} M_{s_{i, p}} \boldsymbol{\Phi}(t) \mathbf{D}_{\phi} \hat{\mathbf{x}}_{p}^{v}(t)+\sum_{p=1}^{n} B_{s_{i, p}} \boldsymbol{\Phi}(t) \hat{\mathbf{x}}_{p}^{v} \\
& +\sum_{p=1}^{n} G_{s_{i, p}} \boldsymbol{\Phi}(t) \hat{\mathbf{x}}_{p}^{q}+\sum_{p=1}^{n} \int_{-\infty}^{t} K_{\mathrm{rad}, s_{i, p}}(t-\tau) \\
& \times \boldsymbol{\Phi}(\tau) \hat{\mathbf{x}}_{p}^{v} d \tau-f_{\mathrm{wave}, s_{i}}(t)-\sum_{p=1}^{2} F_{p, s_{i, k}} \boldsymbol{\Phi}(t) \hat{\mathbf{u}}_{p}(45)
\end{aligned}
$$

where $i=1, \ldots, n$, and $M_{s_{i, p}}, B_{s_{i, p}}, G_{s_{i, p}}, K_{\mathrm{rad}, s_{i, p}}$ and $F_{p, s_{i, k}}$ are the elements of the matrices $\mathbf{M}_{s}, \mathbf{B}_{s}, \mathbf{G}_{s}, \mathbf{K}_{\mathrm{rad}, s}$ and $\mathbf{F}_{p, s}$ respectively. PS methods are used to compute the coefficients $\hat{\mathbf{x}}_{i}^{q}, \hat{\mathbf{x}}_{i}^{v}$ and $\hat{\mathbf{u}}_{p}$ that minimize the residuals (41)-(43) and (44) and (45) for the DAE and ODE formulations, respectively [13]. The PS methods force the residuals of the equations of motion to be zero at a certain number of points in time $t_{k}$, called nodes. If the number of nodes is $N_{c}$, then a nonlinear system of $(2 \times 6 N+m) \times N_{c}$ and $2 \times n \times N_{c}$ equations is solved for the DAE and ODE formulations, respectively. The number of nodes depends on multiple factors, including the order of the expansion $M$ [14].

The optimal control problem defined by the maximization of the cost function (27), subject to the dynamic constraints (1)-(3) and (16) and (17) for the DAE and ODE formulations, respectively, is transformed into a finite dimensional optimization problem with cost function (35), and dynamic constraints (41)-(43) and (44) and (45) for the DAE and ODE formulations, respectively. Additional inequality constraints on the applied PTO torques and relative pitch velocity between the bodies can 


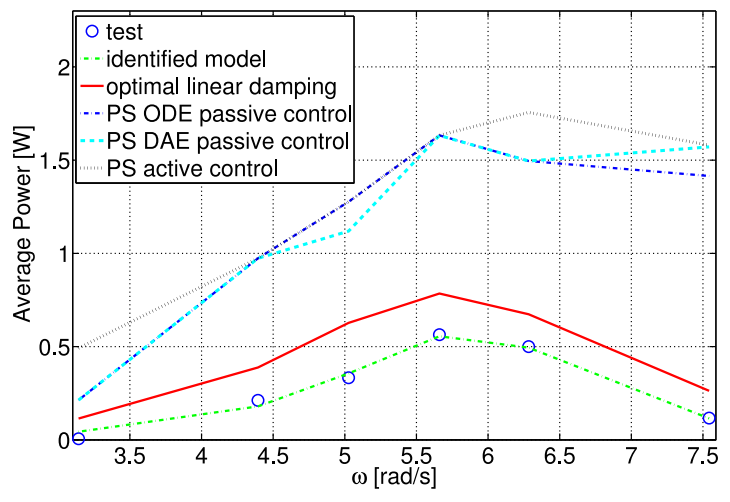

Fig. 2. Comparison between the average power given by the tank data, dynamic model, optimal linear damping control, PS ODE and DAE passive control and PS active control for different frequencies of a regular wave of amplitude $A=2 \mathrm{~cm}$.

also be considered in the optimization problems:

$$
\mathbf{g}\left(\hat{\mathbf{x}}_{i}^{q}, \hat{\mathbf{x}}_{i}^{v}, \hat{\mathbf{u}}_{p}\right) \leq 0
$$

with $i=1, \ldots, 6 N$ and $i=1, \ldots, n$ for the DAE and ODE formulations, respectively, and $p=1,2$.

\section{RESULTS}

A specific three-body hinge-barge device was tested in a wave tank using facilities of the U.S. Naval Academy, Annapolis [15]. The dimensions of the barges are as follows:

1) body 1: length $=0.68 \mathrm{~m}$, width $=0.4 \mathrm{~m}$ and height $=$ $0.1 \mathrm{~m}$

2) body 2: length $=0.28 \mathrm{~m}$, width $=0.4 \mathrm{~m}$ and height $=$ $0.15 \mathrm{~m}$

3) body 3: length $=1 \mathrm{~m}$, width $=0.4 \mathrm{~m}$ and height $=0.1 \mathrm{~m}$.

The dynamic model for the device is identified and validated in [11].

\section{A. Regular Waves}

The power dissipated by the PTO systems was recorded for a series of regular wave tests performed for a range of frequencies $\omega$ from $3.14 \mathrm{rad} / \mathrm{s}$ to $7.54 \mathrm{rad} / \mathrm{s}$ and direction of the waves along the longitudinal direction of the device. In Fig. 2, the dynamic model shows a good agreement with the tank data in terms of average absorbed power.

An alternative strategy to PS methods for the control of the device is to consider a linear model in the frequency domain, and compute the optimal damping coefficients of the PTOs that maximizes the energy absorption at each frequency of the incoming wave [16]. In Fig. 2, the average power given by the dynamic model with optimal linear damping coefficients for the PTOs is shown. In the optimization of the damping coefficients, constraints on the applied PTO torques and relative pitch velocity between bodies are considered.

Next, a passive controller (i.e. only positive power flow from the device) is computed with PS methods, where the torques applied by the PTOs are independent of the relative pitch velocity between the bodies. In Fig. 2, the average absorbed power with a passive controller computed with both DAE and ODE formulations is shown. An active-set algorithm [17] is used for the computation of the PS passive control for both DAE and ODE formulations. As Fig. 2 shows, for both the DAE and ODE formulations, PS passive control significantly outperforms a control strategy based on optimal linear damping coefficients at each frequency of the incoming wave.

For the PS passive control, the average power with the ODE formulation is different from the average power with the DAE formulation. While PS methods gives the same solution to the dynamics of the hinge-barge device [11] for both ODE and DAE formulation, the solution to the optimal passive control problem depends on the formulation used to describe the dynamics of the device. In fact, for the simulation of the linear model of the device with no control applied, the equations of motion in residual form can be rearranged as system of equations where the unknown variables are the expansion coefficients. The number of equations and variables of the system is the same for both DAE and ODE formulations, and its solution is unique provided that the system of equations is nonsingular. For the optimal control of the device, the cost function considered in equation (35) is nonconvex quadratic and, furthermore, for the passive control, the condition of passivity introduces a nonconvex quadratic inequality constraint in the optimization problem [4]. Therefore, the optimal passive control problem can be considered as a nonconvex Quadratically Constrained Quadratic Program [18] and, therefore, a globally optimal solution cannot be guaranteed for both DAE and ODE formulations.

An active controller is also computed with PS methods, where the flow of power is considered to be bi-directional, so that power from the grid can be injected into the device. In Fig. 2, the average absorbed power with an active controller is shown. An active-set algorithm is again used for the computation of the PS active control, for both DAE and ODE formulations. As Fig. 2 shows, in contrast to the optimal passive problem, the solution to the optimal active control problem does not depend on the formulation used to describe the dynamics of the device. Although the cost function in equation (35) is nonconvex quadratic, the active-set algorithm is able to find the global optimal solution to the active control problem. It is important to highlight that the solution for the PS active control, computed with an interiorpoint algorithm [19], depends on the formulation adopted to describe the dynamics of the device. As Fig. 2 shows, the PS active control does not show a significant improvement in term of average power with respect to the PS DAE and ODE passive control. For a certain range of frequencies, from $4.5 \mathrm{rad} / \mathrm{s}$ to $5.5 \mathrm{rad} / \mathrm{s}$, the average power computed by the PS active and passive control is identical, with no benefit in the use of reactive power within the constraints on forces and velocities. The small increase in the average power achievable with PS active control at other frequencies results from the active controller being better able to exploit the operational space using reactive power. If the constraints are relaxed, then the increases in the average power computed with PS active control with respect to the PS DAE and ODE passive control will be more significant. 


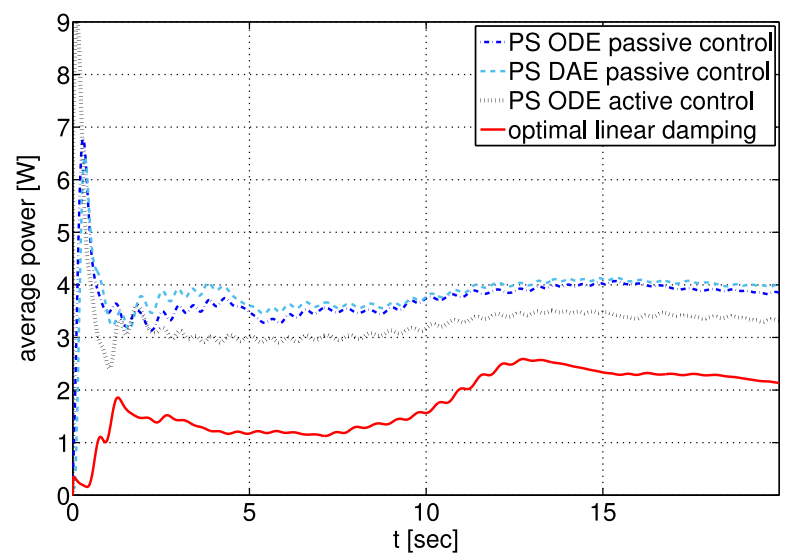

Fig. 3. Time domain comparison of the power absorbed by the hinge-barge device with optimal linear damping control, PS ODE and DAE passive control and PS active control for a polychromatic wave made using JONSWAP spectrum with a significant wave height $H_{s}=15 \mathrm{~cm}$ and significant period $T=$ $1.276 \mathrm{~s}$

\section{B. Irregular Waves}

In Fig. 3, a comparison between the average power absorbed with the optimal linear damping control, PS ODE and DAE passive control, and PS active control is shown for a polychromatic wave over a time horizon of $20 \mathrm{~s}$. The polychromatic wave is from a JONSWAP spectrum with a significant wave height $H_{s}=15 \mathrm{~cm}$ and significant period $T=1.276 \mathrm{~s}$. While the PS active and passive control maximize the average absorbed power over the entire time horizon, the coefficients of dash-pot systems of the optimal linear damping control are set equal to their optimal values at the peak frequency of the spectrum of the incoming wave. An interior-point algorithm is used for the computation of the solution of both PS passive and active control, since the computational time required by an active-set algorithm is excessive. As shown in Fig. 3, the PS passive control outperforms the active control since the interior-point algorithm is not able to compute the global optimal solution of the PS active control.

\section{INTRODUCTION OF CONTROL WEIGHTING}

This section describes a method to obtain a convex cost function for the optimal PS control problem. The following alternative cost function to equation (27) is considered

$$
J=\frac{1}{T} \int_{0}^{T}\left(\mathbf{v}^{T} \mathbf{F}_{p} \mathbf{u}-r \mathbf{u}^{T} \mathbf{u}\right) d t
$$

where $r$ is a weighting parameter. The cost function in equation (47) is composed of the sum of two terms: The average power absorbed by the PTOs and the squared norm of the control vector weighted by the parameter $r$. The parameter $r$ can be physically interpreted as a weight on extreme (squared) values of the control force $u$ and also serves to help to convexify the performance function in equation (47). By substituting the approximated velocities and control torques, defined in equations (29) and (30), respectively, into equation (47), the following cost function is

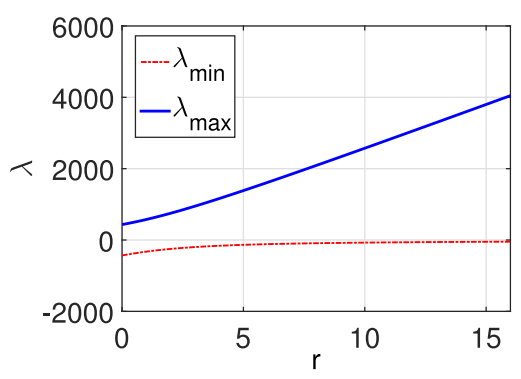

Fig. 4. Minimum and maximum eigenvalue of the matrix $\mathbf{H}$ computed for different values of the weighting parameter $r$

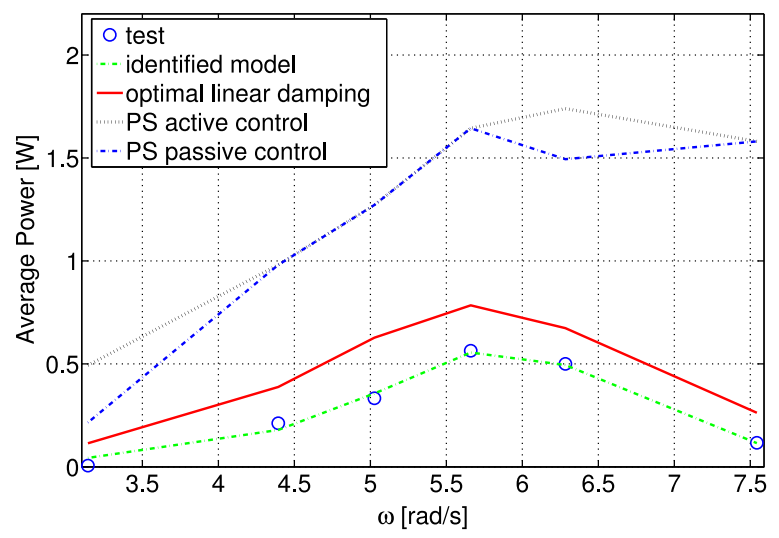

Fig. 5. Comparison between the average power given by the tank data, dynamic model, optimal linear damping control, PS passive control and PS active control with convex cost function for different frequencies of a regular wave of amplitude $A=2 \mathrm{~cm}$.

obtained

$$
\begin{aligned}
J^{M} & =\frac{1}{T} \int_{0}^{T} \mathbf{\Phi}(t)^{T}\left(\mathbf{X}^{V} \mathbf{F}_{p} \mathbf{U}^{T}+r \mathbf{U} \mathbf{U}^{T}\right) \mathbf{\Phi}(t) d t \\
& =\frac{1}{2}\left(\hat{\mathbf{u}}_{1}^{T}\left(\hat{\mathbf{x}}_{2}^{v}-\hat{\mathbf{x}}_{4}^{v}\right)+\hat{\mathbf{u}}_{2}^{T}\left(\hat{\mathbf{x}}_{6}^{v}-\hat{\mathbf{x}}_{4}^{v}\right)-r\left(\hat{\mathbf{u}}_{1}^{T} \hat{\mathbf{u}}_{1}+\hat{\mathbf{u}}_{2}^{T} \hat{\mathbf{u}}_{2}\right)\right) \\
& =\frac{1}{2} \mathbf{x}^{T} \mathbf{H} \mathbf{x}
\end{aligned}
$$

which is a quadratic cost function in the expansion coefficients of the control torques, positions and velocities. The quadratic function in equation (48) is convex if the matrix $\mathbf{H}$ is positive semidefinite, i.e. its eigenvalues are all nonnegative [18]. In Fig. 4, the minimum and maximum eigenvalue of the matrix $\mathbf{H}$ are plotted for different values of the weighting parameter $r$. As shown in Fig. 4, the minimum eigenvalue become nonnegative approximately for values of $r$ greater than 10 .

\section{A. Regular Waves}

In Fig. 5, a comparison between the average power absorbed with the optimal linear damping control, PS passive and active control is evaluated for each frequency of the regular incoming wave. A convex cost function, defined as in equation (48) with $r=10$, is considered for both PS passive and active control. An active-set algorithm is used for the computation of the solution of both optimal passive and active control. 


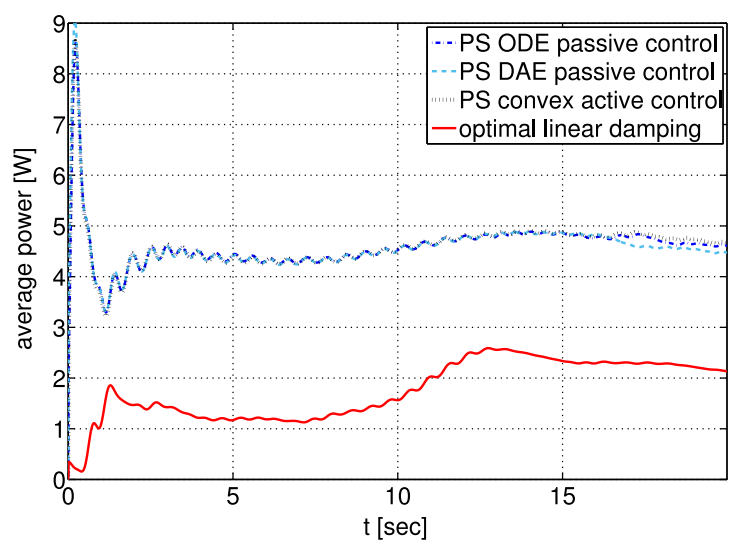

Fig. 6. Time domain comparison of the power absorbed by the hinge-barge device with optimal linear damping control, PS ODE and DAE passive control and PS active control with convex cost function for a polychromatic wave made using JONSWAP spectrum with a significant wave height $H_{s}=15 \mathrm{~cm}$ and significant period $T=1.276 \mathrm{~s}$.

As shown in Fig. 5, both PS passive and active control results are not sensitive to the formulation used to describe the dynamics of the device. Although the PS passive control problem is still not a convex optimization problem due to the passivity condition, the active-set algorithm computes the same solution for both DAE and ODE formulation and, therefore, the global optimal solution is found. For the PS active control problem, both interior-point and active-set algorithm do not depend on the formulation used to describe the dynamics of the device, since the optimization problem is convex.

\section{B. Irregular Waves}

In Fig. 6, a comparison between the average power absorbed with the optimal linear damping control, PS ODE and DAE passive control, and PS active control with convex cost function is shown for a polychromatic wave over a time horizon of $20 \mathrm{~s}$. The polychromatic wave is made using JONSWAP spectrum with a significant wave height $H_{s}=15 \mathrm{~cm}$ and significant period $T=1.276 \mathrm{~s}$. An interior-point algorithm is used for the computation of both PS passive and active controls. As shown in Fig. 6, the PS active control, with a convex cost function, delivers an average power which is greater or equal to the power computed with a PS ODE and DAE passive control. From the comparison between Fig. 3 and Fig. 6, it can be seen that the addition of an extra term of the control action in the cost function yields an increase of the power achievable with a nonconvex cost function for both passive and active control.

However, as shown in Fig. 7, the use of a convex cost function cannot guarantee an average power for the PS active control which is greater than the average power for the PS passive control for all time horizons. Furthermore, for some particular time horizons, the introduction of an extra term of the control action in the cost function penalizes the average power achievable with a nonconvex cost function. In conclusion, a trade-off value for $r$ that ensures the convexity of the cost function, without degrading the absorbed power significantly, must be selected appropriately. The value for $r$ must be re-tuned as the spectral

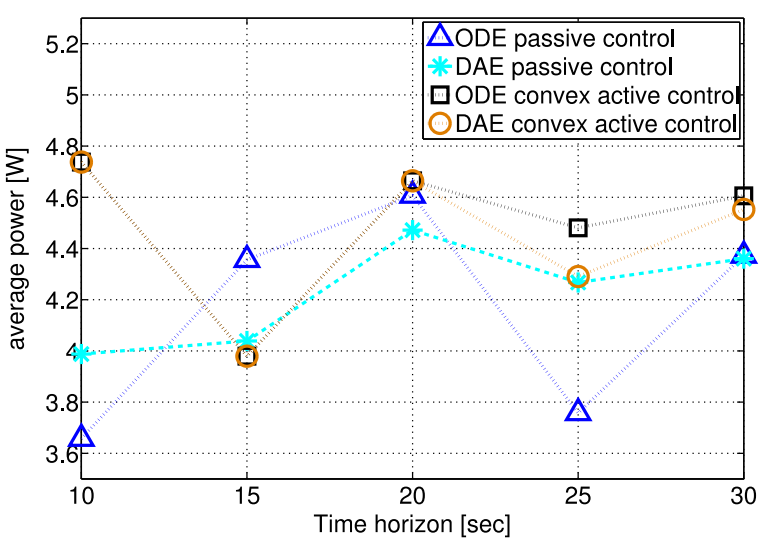

Fig. 7. Average power computed with PS ODE and DAE passive control, PS $\mathrm{ODE}$ and DAE active control with convex cost function for different time horizons of the control problem for a polychromatic wave made using JONSWAP spectrum.

content of the excitation force changes. This is consistent with the use of MPC for wave energy conversion control problems [20].

\section{CONCLUSION}

This paper shows the benefits of PS optimal passive and active control respect to a strategy based on optimal linear passive dampers for the maximization of the energy extracted by a three-body hinge-barge device. In particular, for regular waves, the average absorbed power with PS optimal control is approximately two times greater than the average absorbed power with optimal linear passive dampers around the resonance of the device. For irregular waves, a similar increase of the average power can be achieved with PS optimal control respect to optimal linear dampers. In terms of convexity properties of PS methods, for regular waves, the use of a convex cost function gives an unique solution to both the passive and active control problems which are insensitive to the formulation used to describe the dynamics of the device. However, for irregular waves, the use of a convex cost function does not provide unique solutions to both passive and active control problems and, furthermore, the PS active control cannot guarantee an average power greater than the PS passive control for all the time horizons of the optimal control problem. The results also demonstrate that there is little advantage in using a active controller for a three-body hingebarge device, in terms of absorbed power. Given the marginal benefits of having a reactive controller, considering a realistic PTO efficiency [21], a further reduction of any benefit is expected. Also, the costs involved with the use of a bi-directional PTO can exceed the small increment in the value of absorbed power that can be achieved with a simpler passive PTO.

\section{ACKNOWLEDGMENT}

The authors would like to thank A. Paulmeno and S. E. Mouring from the U.S. Naval Academy for providing the data for the validation of the hinge-barge model. 


\section{REFERENCES}

[1] A. Falcão, "Wave energy utilization: A review of the technologies," Renewable Sustain. Energy Rev., vol. 14, no. 3, pp. 899-918, 2010.

[2] Sea Power Ltd. [Online]. Available: http://www.seapower.ie

[3] R. Yemm, D. Pizer, C. Retzler, and R. Henderson, "Pelamis: Experience from concept to connection," Philosoph. Trans. Roy. Soc. A, vol. 370, pp. 365-380, 2012.

[4] Ø. Y. Rogne, "Numerical and experimental investigation of a hinged 5-body wave energy converter," Ph.D. dissertation, Faculty Eng. Sci. Technol., Dept. Marine Technol., Norwegian Univ. Sci. Technol., Trondheim, Norway, 2014.

[5] J. Hals, J. Falnes, and T. Moan, "Constrained optimal control of a heaving buoy wave-energy converter," J. Offshore Mech. Arctic Eng., vol. 133, pp. $0114011-01140115,2011$.

[6] S. R. K. Nielsen, Q. Zhou, M. M. Kramer, B. Basu, and Z. Zhang, "Optimal control of nonlinear wave energy point converters," Ocean Eng., vol. 72, pp. 176-187, 2013.

[7] R. Genest and J. V Ringwood, "Receding horizon pseudospectral control for energy maximization with application to wave energy devices," IEEE Trans. Control Syst. Technol., 2016, to be published.

[8] C. Canuto, Y. Hussaini, A. Quarteroni, and T. Zang, Spectral Methods: Fundamentals in Single Domains. Berlin, Germany: Springer-Verlag, 2006.

[9] B. Fornberg, A Practical Guide to Pseudospectral Methods. Cambridge, U.K.: Cambridge Univ. Press, 1996.

[10] G. Bacelli and J. V. Ringwood, "Numerical optimal control of wave energy converters," IEEE Trans. Sustain. Energy, vol. 6, no. 2, pp. 294-302, Apr. 2015.

[11] F. Paparella, G. Bacelli, A. Paulmeno, S. E. Mouring, and J. V. Ringwood, "Multibody modelling of wave energy converters using pseudo-spectral methods with application to a three-body hinge-barge device," IEEE Trans. Sustain. Energy, vol. 7, no. 3, pp. 966-974, Jul. 2016.

[12] WAMIT User Manual Version 7.0, Massachusetts Inst. Technol., Cambridge, MA, USA, 2013.

[13] G. Elnagar, M. A. Kazemi, and M. Razzaghi, "The pseudospectral Legendre method for discretizing optimal control problems," IEEE Trans. Automat. Control, vol. 40, no. 10, pp. 1793-1796, Oct. 1995.

[14] I. M. Ross and M. Karpenko, "A review of pseudospectral optimal control: From theory to flight," Annu. Rev. Control, vol. 36, no. 2, pp. 182-197, 2012.

[15] A. Paulmeno, "An experimental analysis of the optimal design conditions for a model scale mccabe wave pump,", Dept. Naval Archit. Ocean Eng., United States Naval Acad., Annapolis, MD, USA, Independent Research in Ocean Engineering Honors Final Report, May 2013.

[16] J. Falnes, Ocean Waves and Oscillating Systems. Cambridge, U.K.: Cambridge Univ. Press, 2002.

[17] P. E. Gill, W. Murray, and M. W. Wright, Practical Optimization. New York, NY, USA: Academic, 1981.
[18] S. Boyd and L. Vandenberghe, Convex Optimization. Cambridge, U.K.: Cambridge Univ. Press, 2004.

[19] R. H. Byrd, J. C. Gilbert, and J. Nocedal, "A trust region method based on interior point techniques for nonlinear programming," Math. Program., vol. 89, no. 1, pp. 149-185, 2000.

[20] G. Li and M. R. Belmont, "Model predictive control of sea wave energy converters-Part I: A convex approach for the case of a single device," Renewable Energy, vol. 69, pp. 453-463, 2014.

[21] R. Genest, F. Bonnefoy, A. H. Clément, and A. Babarit, "Effect of nonideal power take-off on the energy absorption of a reactively controlled one degree of freedom wave energy converter," Appl. Ocean Res., vol. 48, pp. 236-243, 2014.

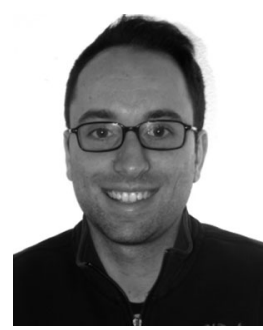

Francesco Paparella received the Laurea Magistrale degree in automation engineering from the Politecnico di Milano, Milan, Italy, in 2012. He is currently working toward the Ph.D. degree at the Centre for Ocean Energy Research, Maynooth University, Maynooth, Ireland. During his thesis he worked on the load sharing optimization of compression stations at the ABB Corporate Research Center, Baden, Zurich. From January 2013 to September 2013, he was a Researcher at RSE S.p.a., Milan working for the development of electrical systems.

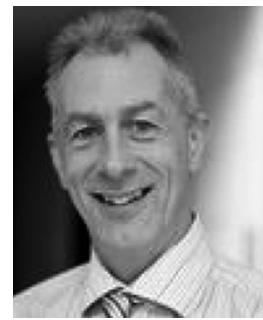

John V. Ringwood (M'87-SM'97) received the Diploma degree in electrical engineering from Dublin Institute of Technology, Dublin, Ireland, in 1981, and the Ph.D. degree in control of steel mills from Strathclyde University, Glasgow, U.K., in 1985. He is currently a Professor of electronic engineering at Maynooth University, Maynooth, Ireland. From 2000 to 2006, he was the Head of the Electronic Engineering Department, Maynooth University. His research interests include time series modeling, wave energy, control of plasma processes, and biomedical engineering. He is a Chartered Engineer and a Fellow of the Institution of Engineers of Ireland. 